\title{
CUADRO DE MANDO PARA EL MONITOREO Y CONTROL DE INDICADORES DE GESTIÓN EN PROCESOS DE INVESTIGACIÓN, INNOVACIÓN Y EMPRENDIMIENTO EN NICARAGUA
}

\author{
MONITORING CHART AND CONTROL OF MANAGEMENT INDICATORS IN THE \\ PROCESSES RESEARCH, INNOVATION AND ENTREPRENEURSHIP IN NICARAGUA
}

\author{
Juan de Dios Bonilla Anduray ${ }^{1}$ \\ Manuel Enrique Pedroza Pacheco²
}

(Recibido/received: 24-Octubre-2019; aceptado/accepted: 3-Diciembre-2019)

\begin{abstract}
RESUMEN: Con el propósito de desarrollar un sistema de Información Gerencial de los indicadores de ciencia, tecnología e innovación para el monitoreo y control de los procesos de investigación, innovación y emprendimiento de las universidades miembros del Consejo Nacional de Universidades (CNU) de Nicaragua, en el periodo 2017-2019, se realizó la investigación con base en el enfoque mixto, que permitió la combinación de métodos cuantitativos y cualitativos, como, la revisión bibliográfica, encuesta, validación de expertos, entrevista, análisis de contenido y cuestionarios de opinión de usuarios. Los resultados reflejan un total de quince categorías para los cuarenta y ocho indicadores organizados en cuatro dimensiones principales financiera, docentes y estudiantes, recursos humanos, procesos de investigación, innovación y emprendimiento, que permiten la toma de decisiones sobre una visión global e integral. Se analizaron los objetivos estratégicos del CNU en las cuatro perspectivas que derivaron en un mapa estratégico de procesos, produciendo un cuadro de mando con los factores claves de éxito, $\mathrm{KPIs}$, los cuales permiten el monitoreo y control del desarrollo de estos procesos. Se desarrolló un sistema gerencial con interfaz gráfica con acceso desde la web desde diferentes dispositivos.
\end{abstract}

PALABRAS CLAVE: Indicadores, Investigación, Innovación, Emprendimiento, Cuadro de mando.

\footnotetext{
${ }^{1}$ Máster. Estudiante de Doctorado DOGCINV, UNAN-Managua.

2 Doctor. Tutor científico. Coordinador DOGCINV UNAN-Managua. Primera Cohorte. 2016-2019. hppedroza@gmail.com.
} 
ABSTRACT: With the purpose of developing a Management Information system of the science, technology and innovation indicators for the monitoring and control of the research, innovation and entrepreneurship processes of the member universities of the National Council of Universities (CNU) of Nicaragua, in the period $2017-2019$, this research was carried out based on the mixed approach, which allows the combination of quantitative and qualitative methods, such as bibliographic review, survey, expert validation, interview, content analysis and user opinion questionnaires. The results reflect a total of fifteen categories for the forty-eight indicators organized in four main dimensions Financial, Teachers and students, Human resources, research processes, innovation and entrepreneurship, which allow decision making on a comprehensive and comprehensive vision. The strategic objectives of the CNU were analyzed in the four perspectives that resulted in a strategic process map, producing a monitoring chart with the key success factors, KPIs, which allow the monitoring and control of the development of these processes. A management system with graphical interface with access from the web from different devices was developed.

KEYWORDS: Indicators, Research, Innovation, Entrepreneurship, Monitoring chart.

\section{INTRODUCCIÓN}

Las herramientas automatizadas de toma de decisiones en la actualidad juegan un papel importante y estratégico en las instituciones, ya que estas necesitan planificar y decidir en base a datos actualizados, pertinentes y correctos, principalmente en lo que se refiere a los procesos claves de su actividad principal, como por ejemplo la investigación y docencia en las universidades. Las instituciones de educación superior en sus planes estratégicos plantean la innovación como eje transversal y presente en cada etapa del desarrollo curricular para la formación de habilidades y capacidades de los futuros profesionales, por tal razón se han creado estructuras organizacionales que atiendan el proceso de innovación y su desarrollo, además de fomentar las iniciativas de proyectos por medio de un programa de fondos concursables.

Por otra parte, el estado de Nicaragua plantea en el plan de Ciencia, Tecnología e Innovación 2010-2013, el desarrollo de un sistema de indicadores de ciencia, tecnología e innovación para fortalecer el proceso de diseño y ejecución de políticas de Ciencia, Tecnología e Innovación y la toma de decisiones de los actores del Sistema Nacional de Investigadores de Nicaragua (Alemán, 2015), como resultado de esta iniciativa se lograron algunas bases en este campo, sin embargo no se automatizó el proceso y como consecuencia sigue presentándose una sub estadística de la producción intelectual y técnica del país.

Debido a lo importante del control y monitoreo de los procesos de investigación, innovación y emprendimiento de las universidades miembros del Consejo Nacional de Universidades de Nicaragua, CNU, el presente estudio permitió el desarrollo de un sistema de información gerencial con informes actualizados y datos presentados de forma visualmente atractivos en forma gráfica y fácil de exportar a diferentes formatos electrónicos. Este sistema será una herramienta 
perteneciente al tipo de Cuadro de Mando Integral que se diseña a partir de los denominados factores claves de éxito o KPls (Key Performance Indicators) para cada institución. De esta manera al contar con indicadores se facilita el control de gestión con un enfoque más anticipado debido a que se centra en las causas que van a explicar los resultados programáticos y financieros futuros.

Para dilucidar cómo los estudios de la innovación, las ciencias y la política se involucran y relacionan, es necesario realizar un abordaje de sus inicios y cómo se condensaron de manera tal que, en el presente se consideran parte fundamental en el momento de formular y ejecutar políticas destinadas al desarrollo. Las principales condiciones coyunturales presentes en la evolución de estas políticas podrían comprenderse a partir de los siguientes casos. Los ejemplos históricos, que hacen que la política tecnológica pueda ser conocida como política científica y la idea de las ciencias se comenzó a concebir como una fuerza productiva que pudo ser priorizada en las economías planificadas (Lara y Bermúdez, 2011).

En el año 2010, las Universidades del CNU desarrollaron un ejercicio tendiente a la construcción de indicadores de Ciencias y Tecnología en el ambiente universitario. De este esfuerzo se obtuvo un producto que en la práctica ha tenido muy pocos frutos, más allá de ser un documento base (Alemán, 2015).

El cuadro de mando integral proporciona a los directivos el equipo de instrumentos que necesitan para navegar hacia un éxito competitivo futuro. Hoy en día, las organizaciones están compitiendo en entornos complejos y, por lo tanto, es vital que tengan una exacta comprensión de sus objetivos y de los métodos que han de utilizar para alcanzarlos (Kaplan y Norton, 2002). El cuadro de mando integral (CMI) traduce la estrategia y la misión de una organización en un amplio conjunto de medidas de actuación, que proporcionan la estructura necesaria para un sistema de gestión y medición estratégica. El CMI sigue poniendo énfasis en la consecución de objetivos financieros, pero también incluye inductores de actuación de esos objetivos.

La construcción de indicadores conlleva una reflexión acerca del proceso social mediante el cual son generadas las actividades de las que éstos dan cuenta, en particular, el desempeño de actores sociales concretos, ubicados en un determinado contexto. Desde esta perspectiva es que ha sido resaltada la importancia de sostener estándares de comparación internacional, sin comprometer la viabilidad de la aplicación de los mismos en los países latinoamericanos.

Por lo anterior, se estableció como objetivo el diseñar un cuadro de mando para el monitoreo y control de los indicadores de gestión de los procesos de investigación, innovación y emprendimiento.

\section{MATERIALES Y MÉTODOS}

De acuerdo con el método de investigación el presente estudio es observacional y según el nivel inicial de profundidad del conocimiento es descriptivo (Piura, 2012). De acuerdo, al tiempo de ocurrencia de los hechos y registro de la información, el estudio es prospectivo, por el período y 
secuencia del estudio es transversal y según el análisis y alcance de los resultados el estudio es analítico (Canales, Alvadrado y Pineda, 1996).

En el ámbito de las ingenierías y las Tecnologías de la Información y la Comunicación (TIC), la presente investigación es un estudio de casos, el cual se fundamenta en la aplicación del Enfoque Mixto, promueve la integración completa, es de carácter explicativo y se orienta por una generalización analítica o naturalista para construir y probar teorías (Hernández, Fernández y Batista, 2014).

Para el desarrollo de la investigación y por sus características particulares, la población objeto de estudio fue definida por 12 Universidades miembros del CNU.

En cuanto al enfoque filosófico, por el uso de los instrumentos de recolección de la información, análisis y vinculación de datos, el presente estudio se fundamenta en la integración sistémica de los métodos y técnicas cualitativas y cuantitativas de investigación, por tanto, se realiza mediante un Enfoque Filosófico de Investigación Mixto (Hérnandez, Fernández y Batista, 2014).

El enfoque de la investigación antes descrito, se sustenta en el Paradigma Socio-Crítico. Esta perspectiva surge como respuesta a las tradiciones positivistas e interpretativas y pretenden superar el reduccionismo de la primera y el conservadurismo de la segunda, admitiendo la posibilidad de una ciencia social que no sea ni puramente empírica ni solo interpretativa. El Paradigma Socio-Crítico tiene como sus principios: 1) Conocer y comprender la realidad como praxis; 2) Unir teoría y práctica (conocimiento, acción y valores); 3) Orientar el conocimiento a emancipar y liberar al hombre; 4) Implicar al docente a partir del autorreflexión. El objetivo produce un cuadro con la definición de las dimensiones principales a mostrar en el cuadro de mando integral para el sistema gerencial de control de gestión de indicadores de ciencia, tecnología e innovación $(\mathrm{CTI})$, así mismo, se cuenta con los informes gráficos correspondientes al comportamiento de los principales indicadores de ciencia, tecnología, investigación y extensión (CTIE) definidos para las universidades miembros del CNU.

\section{RESULTADOS Y DISCUSIÓN}

En el presente estudio se propone un modelo de gestión basado en la visión estratégica del CNU y las correspondientes dimensiones que se plantean en el modelo adaptado para el sector educativo, teniendo como resultado lo que se muestra en la figura 1. 


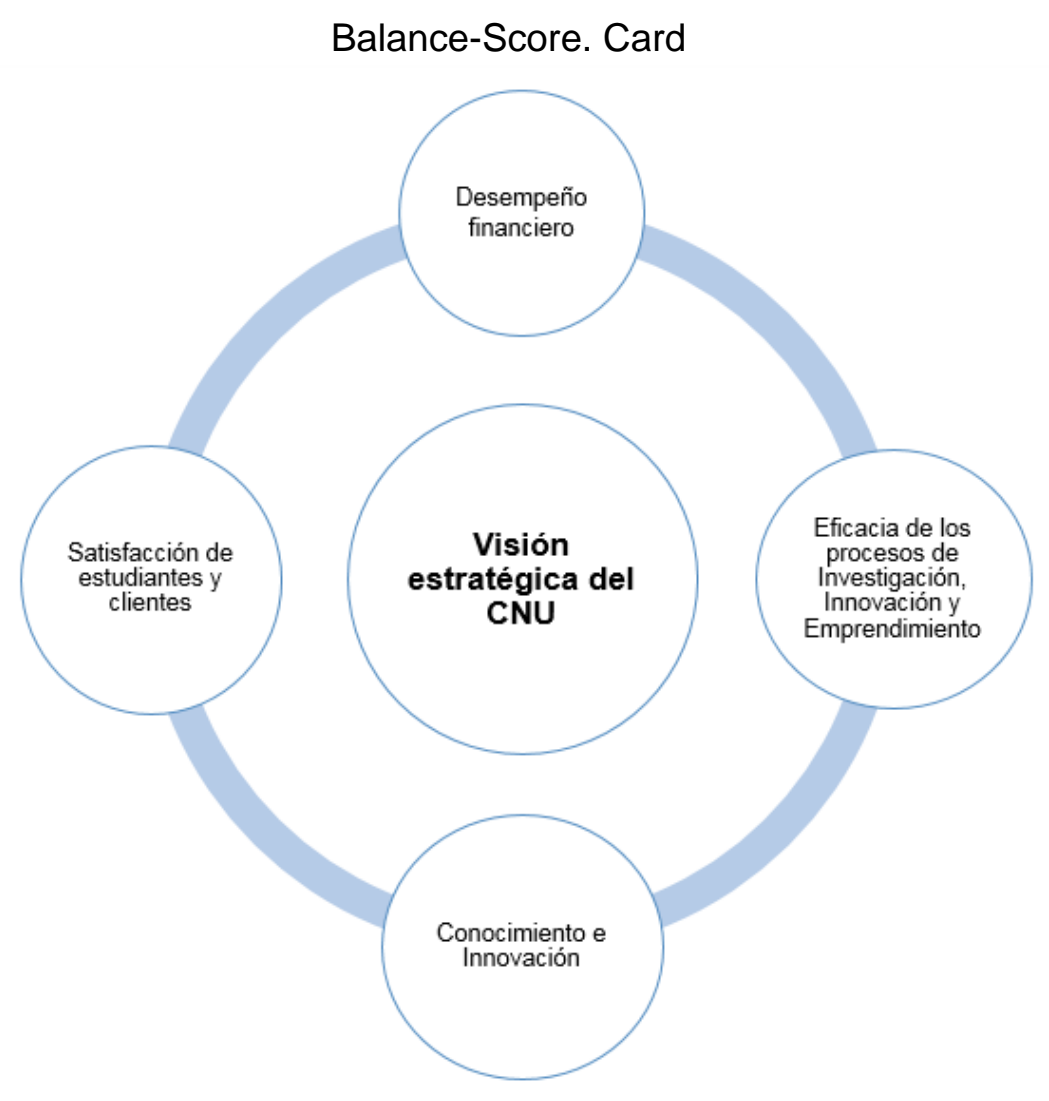

Figura 1. Balanced Card.

En el presente estudio se aplicó las dimensiones de la cultura empresarial al sector educación de la siguiente forma:

1. La dimensión financiera equivalente a presupuesto en el caso de la educación superior.

2. La perspectiva clientes equivale a la perspectiva de docentes y estudiantes beneficiados del servicio de formación del sistema nacional de educación.

3. La perspectiva de procesos internos corresponde a los procesos en estudio como son investigación, innovación y emprendimiento en el nivel universitario.

La perspectiva infraestructura se corresponde a la estructura de recursos humanos con las que se desarrollan los procesos de gestión universitarios.

En toda institución las habilidades de sus empleados es un factor muy importante para el logro de los resultados esperados desde el punto de vista estratégico, en este caso la dimensión Recursos humanos propone dos vías generales respecto al personal encargado de la gestión de los procesos de investigación, innovación y emprendimiento, el fortalecimiento de los conocimientos y habilidades del personal a cargo de los procesos en estudio, que permita conocer las gestiones y los detalles necesarios a nivel procedimental de estos, logrando un funcionamiento eficaz de las solicitudes de información relacionadas a las actividades claves de

El Higo Revista Científica / Volumen 09.No.01, pp. 78-88 / Diciembre 2019 
dichos procesos, se ha tomado en consideración el eje número uno del plan estratégico del CNU, relacionado a la calidad y su lineamiento número cuatro del actual plan estratégico.

La segunda estrategia de esta dimensión propone el uso de una herramienta tecnológica adecuada (Sistema de información gerencial) que permitirá el aumento de la eficiencia en la ejecución de los requerimientos de información involucrada en los procesos de investigación, innovación y emprendimiento.

El mejorar la eficiencia de ejecución de los procesos en estudio implica una disminución en el tiempo de gestión por parte de las instituciones y la generación de información abundante en un formato sencillo para el usuario alcanzando con ello, la satisfacción de los docentes participantes en las actividades relacionadas a los procesos involucrados. La disminución del tiempo en la gestión de los procesos de investigación, innovación y emprendimiento permite a los docentes y estudiantes obtener una respuesta rápida a sus solicitudes de información sobre el desarrollo de estos, lo que significa para la institución un menor costo económico de cada solicitud atendida.

El modelo establece cuatro dimensiones principales iniciando el análisis de la parte financiera que es vital en la existencia de cualquier institución de servicio como el caso del CNU, se estableció el objetivo estratégico principal en esta dimensión "Lograr el incremento de actividades relacionadas a los procesos de investigación, innovación y emprendimiento ajustadas al presupuesto planificado", la medición se realizó por medio de dos indicadores principales como son el total de presupuesto asignado a las actividades de los procesos de investigación, innovación y emprendimiento y el total, tiempo dedicado por el personal a las actividades de los procesos de investigación, innovación y emprendimiento, estos indicadores requieren de un KPIs que permite establecer si la dimensión está en los rangos de valores aceptables o requiere de la atención especial de la alta dirección, por lo general la teoría establece que un valor de ejecución por debajo del $50 \%$ se considere en alto riesgo de no cumplirse, entre cincuenta y setenta por ciento de cumplimiento se considera aceptable y valores superiores al $70 \%$ es lo preferente para el cumplimiento del objetivo.

En la dimensión Estudiantes y docentes se plantea el objetivo "fortalecer la participación de los miembros de la comunidad universitaria en las actividades de los procesos de investigación, innovación y emprendimiento en las universidades miembros del CNU", la medición se realizó por medio de dos indicadores principales como son el total de docentes involucrados en las actividades y total, de estudiantes involucrados en las actividades. Estos indicadores requieren de un KPIs que permite establecer si la dimensión está en los rangos de valores aceptables o requiere de la atención especial de la alta dirección, por lo general la teoría establece que un valor de ejecución por debajo del $50 \%$ se considere en alto riesgo de no cumplirse, entre cincuenta y setenta por ciento de cumplimiento se considera aceptable y valores superiores al $70 \%$ es lo preferente para el cumplimiento del objetivo.

La dimensión Procesos se analiza la Investigación, Innovación y Emprendimiento, se plantea el objetivo "Aumentar la eficiencia de los procesos de Investigación, Innovación y Emprendimiento", 
la medición se realizó por medio de dos indicadores principales como son Total, de publicaciones, Total, de programas especiales, Total, de actividades de los procesos, Total, de productos de propiedad intelectual, Total, de Redes y Total, de Convenios, estos indicadores requieren de un KPI que permite establecer si la dimensión está en los rangos de valores aceptables o requiere de la atención especial de la alta dirección, por lo general la teoría establece que un valor de ejecución por debajo del $50 \%$ se considere en alto riesgo de no cumplirse, entre cincuenta y setenta por ciento de cumplimiento se considera aceptable y valores superiores al $70 \%$ es lo preferente para el cumplimiento del objetivo.

En la dimensión Recursos Humanos se plantea el objetivo "Mejorar los conocimientos, habilidades, herramientas y tecnologías usadas por el personal universitario", la medición se realizó por medio de dos indicadores principales como son Total, de personal de apoyo involucrado en actividades de los procesos y Total, de laboratorios para investigación e innovación a nivel CNU, estos indicadores requieren de un KPI que permite establecer si la dimensión está en los rangos de valores aceptables o requiere de la atención especial de la alta dirección, por lo general la teoría establece que un valor de ejecución por debajo del $50 \%$ se considere en alto riesgo de no cumplirse, entre cincuenta y setenta por ciento de cumplimiento se considera aceptable y valores superiores al $70 \%$ es lo preferente para el cumplimiento del objetivo.

El sistema automatizado derivado del cuadro de mando se denomina GECOINIIE, este reconoce cuatro tipos de categorías de usuarios validos en su lógica, tales como Director de Planificación, Director Investigación, Rectorado de Universidades miembros del CNU y Autoridades del CNU, todos ellos interactuando de forma continua con el GECOINIIE, desde la perspectiva particular de su rol ya sean los dos primeros con el ingreso de las entradas como los dos segundos en el seguimiento y control.

EI GECOINIIE es un sistema de servicio a la toma de decisiones y está diseñado para la entrada de valores esperados a los indicadores de los procesos de investigación, innovación y emprendimiento por los usuarios bajo el rol de planificadores, estos valores se ingresarán una vez al principio del año. Además, se realiza la alimentación de los avances en la ejecución por un periodo de fechas definidos por la alta dirección.

La segunda etapa corresponde al seguimiento y control que pueden realizar las autoridades universitarias del avance en cada dimensión planificada por medio de una serie de gráficos dinámicos que representan claramente la relación de lo planificado y ejecutado desde cada universidad. El usuario bajo el rol de autoridades CNU posee autorización de visualizar los diferentes reportes que generalizan los valores de indicadores en las dimensiones contempladas en el modelo del estudio y que permiten tener una visión integral y globalizada del comportamiento en las universidades miembros del CNU en las variables principales del cuadro de mando, el que se despliega brindando mayor detalle de una forma fácil y desde cualquier dispositivo. 
El sistema utiliza una arquitectura Cliente / Servidor, entiéndase Cliente a diferentes dispositivos como son las computadoras personales, Smartphone o Tablet, los cuales utilizan un software conocido como navegador para comunicarse a través de internet hacia los servidores web del CNU donde se encuentra alojada la aplicación web que se comunicará con el servidor de bases de datos, en este estudio se utilizó el MySql de software libre.

En el establecimiento del cuadro de mando para realizar el monitoreo y control de los indicadores de gestión de los procesos en estudio, se ejecutó como primer paso la creación de un mapa estratégico, para lo cual se utilizó la misión, visión, objetivos y ejes estratégicos del CNU relacionados a la ciencia, tecnología e innovación. En la misión del CNU se establece que el subsistema de educación nicaragüense tiene como finalidad la generación y difusión de conocimientos a través de la investigación, la extensión y la innovación con calidad, pertinencia e interculturalidad, con el fin de aportar a la sociedad nicaragüense un talento humano responsable, ético, solidario, reflexivo y crítico, lo que justifica la importancia del desarrollo de los procesos de investigación, innovación y el emprendimiento para cada universidad. Así mismo, en la visión, el CNU establece el uso de los conocimientos y saberes locales, las tecnologías de la información y la comunicación, para contribuir al desarrollo humano integral, científico y tecnológico del país.

Entre las principales actividades de los procesos se encuentran la Jornada universitaria de desarrollo científico, los congresos docentes, las investigaciones realizadas por estudiantes y docentes de los niveles permitidos por la oferta académica de las universidades, la elaboración de proyectos innovadores, el concurso de fondos para la innovación, el desarrollo de prototipos y talleres de capacitación en el tema de innovación, el uso del método canvas, la elaboración de planes de negocios, estas contribuyen a la transferencia del conocimiento de la academia a la sociedad. Lo anterior permitió la identificación de los docentes, estudiantes, administradores, directores de planificación e investigación de las universidades como actores principales en el desarrollo y ejecución de los procesos en estudio.

El diseño del tablero de control responde al modelo de cuatro dimensiones principales para el CNU, desarrollándose en la dimensión financiera el objetivo correspondiente al incremento de actividades relacionadas a los procesos en estudio que se ajusten al presupuesto planificado, para alcanzar un monitoreo constante de la evolución de las actividades se definieron un conjunto de indicadores para los cuales se establecen metas específicas de cumplimiento conocidas como $\mathrm{KPIs}$, en este caso se estudia el total de presupuesto asignado a las actividades de los procesos de investigación, innovación y emprendimiento y el Total, de tiempo dedicado por el personal a las actividades de los mismos procesos.

En el modelo diseñado para el CNU se establecen como valores generales de los KPIs la siguiente escala: valores menores al $50 \%$ de lo planificado es de atención primordial de la alta dirección, entre 50 y $70 \%$ del valor previsto existe un mediano riesgo de cumplimiento y los valores superiores al $70 \%$ es de posible alcance sin riesgo. 
Por parte de la dimensión Estudiantes y docentes del modelo se desarrolló el objetivo correspondiente al fortalecimiento de la participación de los miembros de la comunidad universitaria en las actividades propias de los procesos en estudio de las universidades, con ello se garantiza lo expresado en la visión referente al impacto social y la inclusión, derivado de esta dimensión se encuentran los indicadores referentes al Total de docentes y total de estudiantes involucrados en las actividades de investigación, innovación y emprendimiento.

El cuadro de mando se implementa en un sistema gerencial de monitoreo y control de indicadores en los procesos de investigación, innovación y emprendimiento bajo una línea gráfica minimalista que permite el acceso a la aplicación desde dispositivos de diferentes dimensiones como son los smartphones y las tabletas. La interfaz de acceso a la aplicación utiliza el método de seguridad usuario y contraseña, donde se consideran validos cinco roles de usuarios correspondientes a los actores claves del modelo de balanced score propuesto para alcanzar la visión del CNU.

La aplicación web despliega diferentes menús en dependencia del rol al que está vinculado el usuario ingresado, teniendo más privilegios el rol administrador de la aplicación, cuya función es velar por la integridad de la base de datos y la alimentación inicial del sistema, los usuarios con el rol de dirección de planificación son los encargados de suministrar los valores anuales planificados para las funciones relacionadas a la investigación, innovación y el emprendimiento. Los directores de investigación son responsables del ingreso del avance en los procesos y cuentan con un módulo que les permite la creación de formularios en línea capaces de recopilar los datos en cualquier sede para generar los valores globales de la ejecución de las actividades. Mientras tanto, los usuarios con el rol de rectorado pueden obtener información en el momento que deseen de los indicadores de CTI en su universidad y comparar el comportamiento de ellos en los años anteriores.

\section{CONCLUSIÓN Y RECOMENDACIÓN}

Se diseñó un cuadro de mando formado por cuatro dimensiones correspondientes a las perspectivas establecidas en el modelo de gestión, estableciéndose las medidas porcentuales y de valor absoluto para los indicadores que permiten un monitoreo y control en tiempo real de los valores en cada indicador por medio de la aplicación web correspondiente, reflejando el resultado con una señalización gráfica de fácil interpretación.

Se recomienda la revisión y seguimiento periódico de los indicadores principales para el control de los procesos, formando una cultura de evaluación de calidad en la comunidad universitaria. Así como, la aplicación web asociada al cuadro de mando se puede actualizar para el incremento de sus potencialidades a nivel de otras instituciones, completando la relación del modelo I + D+ i. 


\section{AGRADECIMIENTOS}

A las Autoridades Superiores de la Universidad Nacional Autónoma de Nicaragua, Managua, quienes creyeron en mí y me dieron el apoyo de tiempo y material necesarios para finalizar estos estudios.

A mi tutor de Tesis doctoral en el Programa DOGCINV, el Dr. Manuel Enrique Pedroza Pacheco, por su asesoramiento científico amplio y profundo, así como por sus sabios consejos que me guiaron correctamente para concluir con éxito mi doctorado en el Programa DOGCINV.

Le agradezco especialmente al programa de Fondos para la Investigación de la UNAN Managua, el cual designó recursos para el presente estudio, facilitando en gran manera su culminación exitosa, de igual forma a la comunidad educativa de las diferentes universidades miembros del $\mathrm{CNU}$, que participaron en el desarrollo de este sistema gerencial, principalmente a los vice rectores y directores de investigación y extensión, quienes siempre estuvieron dispuestos a colaborar con su experiencia y conocimiento en el desarrollo de los procesos de investigación, innovación y emprendimiento que tuvo como fin la mejora de la calidad de la educación superior.

\section{BIBLIOGRAFÍA}

Alemán Zeledón, F. S. (2015). Construcción del sistema de indicadores de ciencia, tecnología e innovación en Nicaragua. Managua, Nicaragua.

Canales, F. H., Alvadrado, E., y Pineda, E. (1996). Metodologuia de la investigacion.

Hernández, R., Fernández, C., y Batista, P. (2014). Metodología de la Investigación. Mc Graw Hill.

Kaplan, R. S., y Norton, D. P. (2002). Cuadro de Mando Integral. Barcelona, España: Gestion 2000.

Lara Rodríguez, J. S., y Bermúdez Guerrero, J. I. (2011). Perspectiva de la política de innovación y su monitoreo en la Unión Europea, 2010-2020. Revista Finanzas y Política Económica, 105-125.

Piura, Julio.(2012). Metodología de la investigación científica/Un enfoque integrador. 7ạ. Ed.

Valle, O., y Rivera, O. (2013). Monitoreo e indicadores. Guatemala: Organización de estados Iberoamericanos. 


\section{SEMBLANZA DE LOS AUTORES}
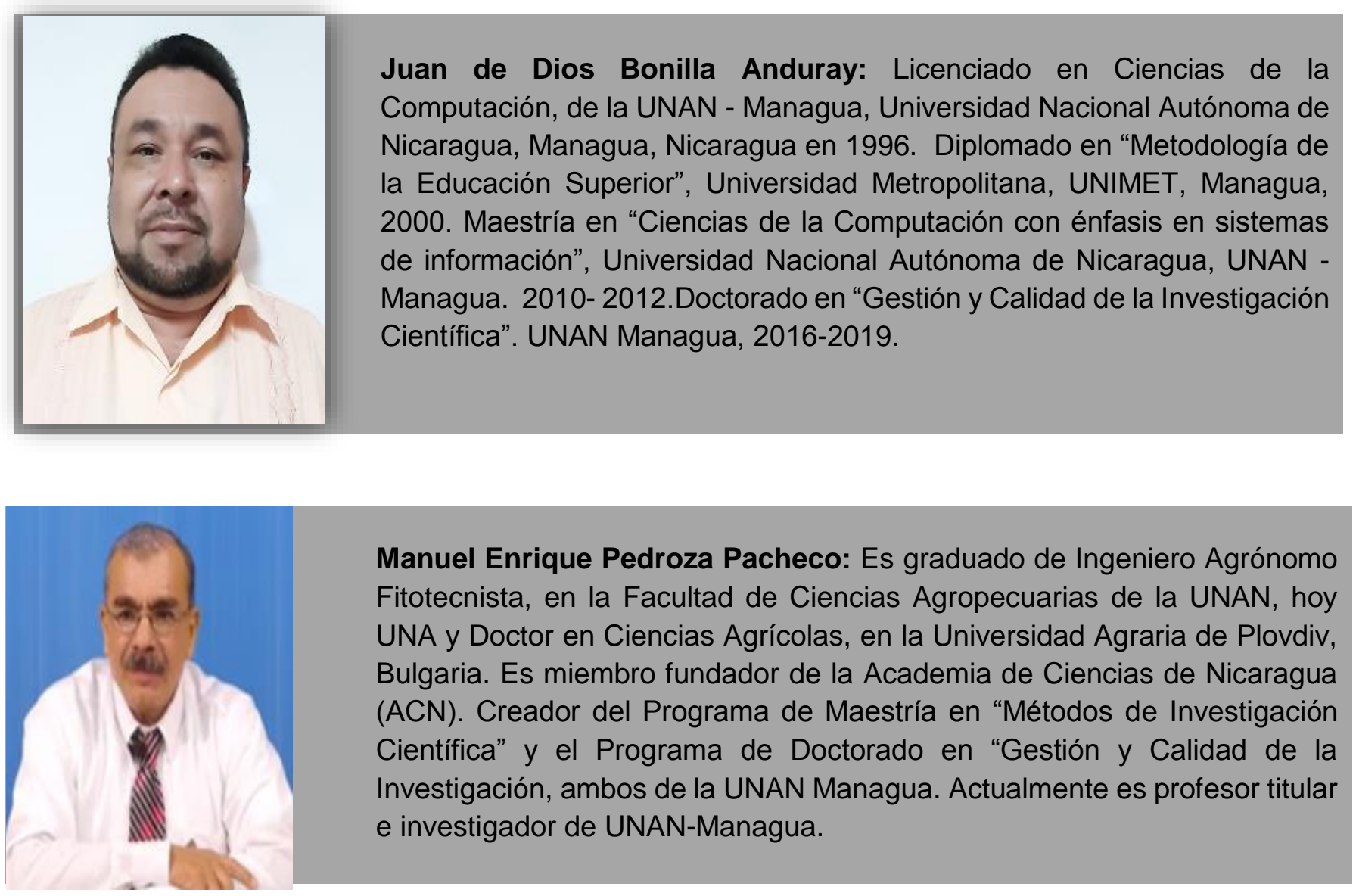